\title{
Acceso léxico y escalaridad: una aproximación experimental al acceso de adjetivos fuertes a partir de adjetivos débiles en español ${ }^{*}$
}

Lexical access and scalarity: an experimental approach to the access from weak to strong adjectives in Spanish

Ittay Gil Carrillo Universidad Nacional Autónoma de México, Escuela Nacional de Lenguas, Lingüistica y Traducción ittaygil@enallt.unam.mx 


\section{Resumen}

Recientemente, algunos estudios han puesto en cuestión la hipótesis de uniformidad - que afirma que un conjunto amplio de escalas de Horn da lugar, si la información contextual lo permite, a implicaturas escalares (IE). Estas investigaciones han mostrado que escalas pertenecientes a diversas categorías gramaticales exhiben muy distintas frecuencias de derivación de IE. La idea es que el uso del elemento débil no garantiza la derivación de una IE. Entre algunos de los fenómenos que explican esta fluctuación está la distancia semántica. Sin embargo, poco se sabe sobre el papel de fenómenos léxicos-cognitivos, como el acceso léxico, en la tendencia a la derivación variable. Aquí se presenta un estudio experimental basado en una tarea de decisión léxica a partir del paradigma de primado enmascarado. El objetivo es dilucidar en qué medida el elemento débil de una escala adjetival está relacionado con el elemento fuerte en términos de acceso léxico. Los resultados muestran que para ciertos casos la exposición al elemento débil no siempre activa significativamente al elemento fuerte, lo cual podría afectar la frecuencia de derivación de IE, al menos en el caso de escalas adjetivales en español.

Palabras clave: adjetivos; antónimos; acceso léxico; implicatura escalar; efecto de primado

\section{Abstract}

Some recent studies have questioned the so-called uniformity hypothesis - a principle stating that Horn scales give raise, if context allows it, to scalar implicatures (sI). These studies have shown that different scales from diverse grammatical categories will give rise to a wide range of derivation frequencies of sI. The idea is that the use of a weak element does not guarantee the derivation of an SI. Among some of the phenomena that explain this fluctuation is semantic distance, for example. Nevertheless, little is known about the role of certain lexical and cognitive phenomena such as lexical access in the derivation process. I present here an experimental study based on a lexical decision task using a masked priming effect. The aim of the study was to elucidate the extent to which the weak element of an adjectival scale can activate the strong one in terms of lexical access. The results show that in certain cases, exposure to the weak element does not always activate the strong one, which could eventually affect the frequency of derivation of sI, at least in the case of adjectival scales in Spanish.

Keywords: adjectives; antonyms; lexical access; scalar implicature; priming effect 


\section{Introducción}

Uno de los fenómenos más explorados en la literatura especializada en pragmática es la implicatura escalar (IE). Se trata de un tipo especial de inferencia pragmática ${ }^{1}$ cuya derivación depende en gran medida del uso, por parte del hablante, de un ítem léxico específico, un elemento escalar, de donde deriva su nombre. ${ }^{2}$ En (1a) se muestra un enunciado donde el uso del cuantificador existencial puede dar lugar a la derivación de una IE como (1b).
(1) a. Algunas manzanas se pudrieron.
b. No todas las manzanas se pudrieron.

$\mathrm{O}$, en el caso de (2a), donde el uso de la disyunción también es susceptible de detonar una IE como (2b).

(2) a. Matías se pintó el pelo de rojo o azul.
b. Matías no se pintó el pelo de rojo y azul.

Tradicionalmente, se entiende que las IE son derivadas a partir de la observación del principio de cooperación griceano y, en específico, de la máxima de cantidad; es por ello que en realidad se trata de un tipo especial de implicatura de cantidad. Sin embargo, su sello característico es justamente la presencia del elemento escalar en la enunciación. Como muestran los ejemplos (1a) y (2a), el uso de

* Agradezco al proyecto "Términos numéricos e implicatura escalar" (DGAPA-PAPIIT IN401415) por el financiamiento recibido para realizar este estudio. También agradezco a Carmen Curcó, Teresa Peralta y Gala Villaseñor por la excelente retroalimentación recibida.

1 En general es aceptado que la implicatura escalar es una inferencia sintética y por tanto cancelable, es decir, un fenómeno pragmático y no semántico o lógico. Sin embargo, hay quienes sostienen que en realidad es un fenómeno gramatical. Véase Chierchia (2004), por ejemplo.

2 En §2 se expone en detalle la noción de escala de Horn y cómo esta se relaciona con la derivación de implicaturas. 
elementos específicos como algunas y o por parte de un hablante es en principio lo que podría autorizar al oyente a derivar que el contenido equivalente con el cuantificador universal todas o la conjunción $y$ no son el caso. Lo interesante de este tipo de elementos es que pueden formar, junto con otros elementos de igual categoría gramatical, un conjunto ordenado en términos de la cantidad de información que son capaces de transmitir, del cual surgirá una relación lógica entre al menos dos elementos, en dicha correspondencia el elemento informativamente más fuerte entrañará lógicamente al más débil. ${ }^{3}$ En el caso de nuestros ejemplos, los cuantificadores forman la escala $<$ algunos, todos $>$ y, las conectivas, la escala $<0$, $\mathrm{y}>$, donde el elemento de la derecha siempre es lógicamente más fuerte que el de la izquierda y por tanto lo entraña.

A partir del trabajo inaugural de Horn (1972; 1992; 1996), se ha dado por sentado que estas escalas existen en las lenguas y que en general el uso del elemento más débil de la escala da lugar a IE. Sin embargo, hay dos cuestiones que requerirían mayor análisis, una directamente derivada de la otra: primero, que la mayoría de conclusiones teóricas al respecto proceden del estudio de IE derivadas a partir de la escala cuantificacional o bien de la escala de conectivas, y segundo, que no se sabe por consiguiente qué sucede con otros elementos léxicos con características similares, o si su uso da pie a la generación de IE; por ejemplo, si el uso de un adjetivo gradual débil como cansado puede dar lugar a la lectura acotada superiormente de no exhausto. Esto resulta crucial, pues el comportamiento de unos cuantos elementos lingüísticos no justificaría la existencia de una clase especial de implicatura, como la escalar. En todo caso, estos ejemplos podrían explicarse simplemente como casos de implicaturas de cantidad. Para ilustrar lo que sucede con elementos distintos a los cuantificadores, por ejemplo, obsérvese ahora (3).

3 Entrañamiento entendido aquí como equivalente a la noción de implicación lógica. $\beta$ entrañará $\alpha$ sii no existe ninguna condición en la que $\beta$ sea verdadera y $\alpha$ no lo sea. 
(3) a. El futbolista está cansado.

b. El futbolista no está exhausto.

De la misma forma que los cuantificadores o las conectivas, algunos adjetivos graduales pueden ser ordenados en términos de informatividad y constituir una escala. En el caso de (3), los adjetivos cansado y exhausto ${ }^{4}$ sin embargo, y a diferencia de los ejemplos (1) y (2), no parece tan evidente que (3b) pueda derivarse de (3a).

Respecto a este punto, recientemente van Tiel, van Miltenburg, Zevakhina y Geurts (2016) abordaron directamente la cuestión de la diversidad escalar y a partir de un estudio empírico pusieron a prueba la capacidad de distintas escalas, provenientes de cinco categorías gramaticales, de dar pie a la derivación de IE. En dicho estudio analizaron 32 escalas adjetivales (entre otras más provenientes de distintas categorías gramaticales) con el objetivo de saber si el uso de los términos débiles detonaba la inferencia de que los fuertes no aplicaban, es decir, detonaban una IE. Para medir esto, se les preguntó explícitamente a los participantes si a partir de un enunciado de la forma " $\alpha$ es el caso" inferirían que " $\beta$ no es el caso". Los autores compararon los resultados a partir de dos condiciones; la primera, que llamaron "más neutral", tenía por sujeto del enunciado un pronombre, y la segunda, designada "menos neutral", cuyo sujeto era una frase nominal, como lo muestran la Figura 1 y 2 (van Tiel et al., 2016).

En líneas generales, este estudio dejó ver una gran variación en el comportamiento de las escalas en cuanto a la tasa de derivación de IE que propician. Como lo muestra la gráfica en la Figura $3,{ }^{5}$ la tasa de derivación de IE a partir de adjetivos distintos es muy variable, exhibiendo frecuencias que van desde $100 \%$ hasta $4 \%$.

4 En el caso del ejemplo, exhausto entraña lógicamente a cansado.

5 Gráfica generada por el autor que integra los datos presentados en van Tiel et al. (2016) concernientes a las escalas adjetivales, de interés para el presente estudio. 
John says:

She is intelligent.

Would you conclude from this that, according to John, she is not brilliant?

\section{$\square$ Yes $\square$ No}

Figura 1. Ejemplo de reactivo más neutral con pronombre (van Tiel et al., 2016)
John says:

$$
\text { This student is intelligent. }
$$

Would you conclude from this that, according to John, she is not brilliant?

$$
\square \text { Yes } \square \text { No }
$$

Figura 2. Ejemplo de reactivo menos neutral con frase nominal (van Tiel et al., 2016)

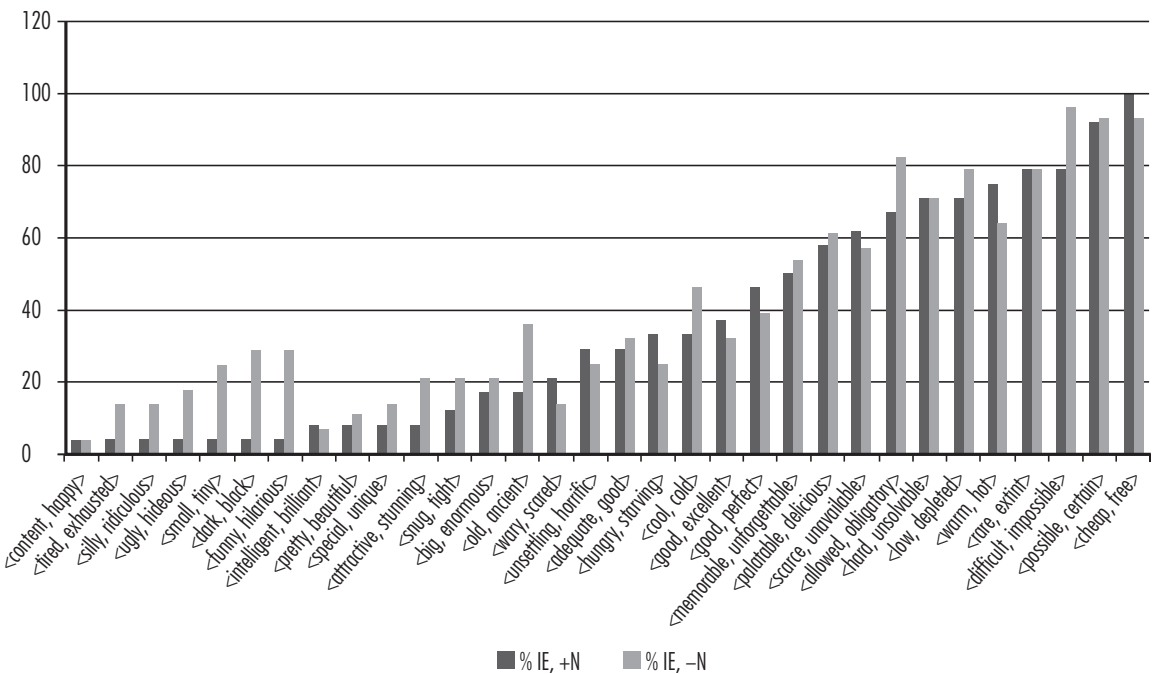

Figura 3. Porcentajes de derivación de IE a partir de 32 escalas adjetivales

A pesar de considerar varios criterios lingüísticos en cuanto a las escalas se refiere - disponibilidad, frecuencia de uso, clase gramatical, parentesco semántico, etc.- - para dar cuenta de sus resultados, ningún criterio pudo explicar la variación ni las enormes diferencias que muchas de ellas presentaron entre sí. Es decir, no resulta claro por qué conjuntos ordenados de elementos que califican por sus características como escalas adjetivales pueden diferir 
tanto en su capacidad para dar pie a IE. O, para regresar a los ejemplos aquí vistos, por qué el uso de un cuantificador débil facultaría al oyente a derivar una IE con mucha más frecuencia que el uso de un adjetivo gradual débil como cansado.

Uno de los objetivos de esta investigación es responder la pregunta: ¿qué hace en principio que una escala sea diferente de otra? A diferencia de estudios precedentes, donde el objetivo es investigar el fenómeno de la IE, sea a partir de escalas distintas o no, se indaga el fenómeno de las escalas mismas y de las diferencias que exhiben entre sí. Para ello, se toma como parámetro de distinción central entre las escalas el acceso léxico, entendido como el grado de activación que presenta un elemento fuerte a partir de uno débil en una escala de Horn. Se presenta un experimento basado en una tarea de decisión léxica con efecto de primado y se reportan los resultados obtenidos para un grupo de 19 escalas adjetivales. La conclusión general es que efectivamente existe una diferencia en cómo los elementos léxicos provenientes de una escala son susceptibles de activar a otros elementos y así facilitar su acceso. Este rasgo, el acceso léxico, podría explicar el comportamiento tan variado de las escalas que ha sido reportado en otros estudios.

\section{Escalas de Horn}

A grandes rasgos, se entiende que una escala de Horn es un conjunto de elementos léxicos pertenecientes a la misma categoría gramatical, que están lexicalizados y que se encuentran ordenados de acuerdo a su grado de informatividad en alguna dimensión conceptual. Los conjuntos ordenados en (4) son algunos ejemplos de escalas de Horn.

(4) a. <algunos, todos>

b. $<0, y>$

c. <grande, enorme>

d. <empezar, terminar> 
Un conjunto ordenado será una escala de Horn si, y solamente si, dicho conjunto contiene al menos dos elementos $\alpha$ y $\beta$, donde $\beta$ es más informativo que $\alpha$. Un elemento $\beta$ será más informativo que uno $\alpha$ si, y solamente si, existe una relación de entrañamiento o implicación lógica que va de $\beta$ a $\alpha$. En otras palabras, si se toma como ejemplo (4c), se observa que todo lo que es enorme también es grande, pero no al contrario. Así, las entidades que comparten la característica de ser enormes constituyen un subconjunto propio del conjunto de entidades que tienen como propiedad ser grandes. Horn (1972) fue el primero en notar esta propiedad de algunos conjuntos ordenados, por lo que comúnmente se les conoce como escalas de Horn. Estas pueden considerarse como un elemento semántico implicado en el método tradicional griceano de derivación de implicaturas de cantidad, pues imponen condiciones sobre lo que podría derivarse y lo que no. En otras palabras, restringen a un número finito las posibles alternativas a partir de las cuales se generan implicaturas de cantidad. Retomando el enunciado en (1a), repetido aquí como (5), puede observarse que de las tres proposiciones en (6), solo (6a) y (6b) contarían como verdaderas alternativas de (5) y por tanto como posibles candidatos a partir de los cuales derivar IE.

(5) Algunas manzanas se pudrieron.

(6) a. Muchas manzanas se pudrieron.

b. Todas las manzanas se pudrieron.

c. Solo algunas manzanas se pudrieron.

Aunque (6c) es informativamente más fuerte que (5) y por tanto podría suponer una alternativa, es imposible derivar a partir de (5) que No es el caso que solo algunas manzanas se pudrieron. Esto no sucede, sin embargo, con (6a) ni con (6b), pues muchos y todos forman parte de la escala cuantificacional <algunos, muchos, todos $>$, a diferencia de solo algunos. Es en este sentido que las escalas de Horn representan una restricción teóricamente plau- 
sible y que también se considere que la sustitución por alternativas escalares, a partir de la cual puede derivarse una IE, resulte una noción intuitivamente correcta, simple y fácil de formalizar (Geurts, 2010).

\subsection{Variedad escalar}

Si bien las escalas de Horn parecen una solución teóricamente elegante a la proliferación de inferencias, no se encuentran libres de problemas, ya que no todo lo que es susceptible de ordenarse en términos de informatividad produce los mismos resultados. Al tomar como ejemplo la escala de sustantivos <animal, gato, siamés $>,{ }^{6}$ parece intuitivamente correcto pensar que si se predica algo de animal, sería probable que surgiera una implicatura como la mostrada en $(7 b)$.

(7) a. Vi un animal en la escalera.

b. No vi un gato en la escalera.

Sin embargo, si ahora se predica algo del segundo elemento de la escala gato, resultaría muy difícil (a menos que un contexto especial lo permitiese) que fuera derivada una implicatura como (8b).

(8) a. Vi un gato en la escalera.

b. No vi un siamés en la escalera.

Estos ejemplos muestran que, aunque teóricamente la noción de escala de Horn parece predecir correctamente la derivación de IE, existen consideraciones adicionales que resultan importantes, como

6 Este conjunto ordenado también puede verse en términos de una relación de hiperonimia/hiponimia, donde animal es hiperónimo de gato y gato de siamés. La relación de hiperonimia/hiponimia es una relación de entrañamiento, donde los hipónimos son subconjuntos propios de los hiperónimos. 
la relevancia. ${ }^{7}$ En este sentido, las escalas de Horn serían representaciones parciales de relevancia contextual (Geurts, 2010). Es decir, el hecho de que el par ordenado < animal, gato $>$ pueda detonar más fácilmente una IE que el par ordenado < gato, siamés $>$ es una prueba de que el primero es una representación más fidedigna de las expectativas que los hablantes tienen en cuanto al uso de ciertas entradas léxicas en contextos no marcados. En otras palabras, el hecho de que (7b) cuente como una posible IE de (7a) se debe a que es probable que al predicar algo de animal el oyente se pregunte sobre la especie de dicho animal, mientras que al predicar algo de gato la pregunta sobre su raza no se genere. Si esto es cierto, la escala <animal, gato> resultaría mucho más relevante que la escala <gato, siamés> y por tanto mucho más susceptible de derivar una IE. ${ }^{8}$

No solo las escalas a partir de hipónimos e hiperónimos resultan controversiales en cuanto a la derivación de IE a la que dan pie. Existe bastante evidencia empírica que sugiere que los numerales, tratados por la tradición neogriceana como otro ejemplo más de escalas de Horn, ${ }^{9}$ se comportan en realidad de manera muy distinta a otras escalas (Papafragou \& Musolino, 2003; Curcó \& Peralta, 2013; Huang, Spelke \& Snedeker, 2013; Villaseñor, 2017).

Por último, estudios recientes como los reportados por Zevakhina (2012) o van Tiel et al. (2016) han intentado refutar empíricamente lo que llaman el supuesto de uniformidad, según el cual se ha dado por sentado en la literatura que la escala de conectivas y la escala cuantificacional son representativas de toda la clase, en

7 Relevancia entendida aquí no en el sentido técnico sino cotidiano, es decir, no en el sentido de Sperber y Wilson (1995) ni en ningún otro sentido desarrollado por la teoría de la relevancia o posteriores modificaciones (Carston, 1998).

Cf., Geurts (2010).

9 En la tradición neogriceana, los numerales forman la escala $<n_{1}, n_{n}>$ y semánticamente codifican $(\mathrm{n} \leq)$. Así, el uso del numeral tres en un enunciado como Tres invitados llegaron a la fiesta recibiría la lectura Exactamente tres invitados llegaron a la fiesta vía implicatura escalar (Levinson, 1983). Esta postura ya no es sostenida por los expertos. 
el sentido de que su comportamiento en cuanto a la derivación de IE puede generalizarse al resto de las escalas. Sin embargo, y a pesar de que efectivamente sus resultados han puesto en duda dicho supuesto, no existe una explicación satisfactoria que lo justifique.

La razón de dicho comportamiento, sin embargo, podría radicar en la relación que mantienen entre sí los elementos dentro de una misma escala de Horn en términos de acceso, es decir, de si el término escalar fuerte es efectivamente accedido cuando el término escalar débil es utilizado. En la siguiente sección se desarrolla esta idea.

\subsection{Acceso léxico}

Como se ha planteado previamente, para que una IE pueda ser derivada se requiere, en primer lugar, de un principio pragmático ${ }^{10}$ que permita al oyente derivar una inferencia a partir de lo dicho por el hablante y, en segundo, el uso por parte del hablante de un elemento léxico específico cuyas características semánticas lo hagan susceptible de formar una escala de Horn. Dadas estas dos condiciones y un contexto adecuado, una IE podría derivarse. Sin embargo, existe un elemento en dicha explicación que se está obviando, y esto es la naturaleza de la escala misma. Puede afirmarse que la IE es una inferencia con la forma mostrada en (9c).

(9) a. $<\alpha, \beta>$

b. $\phi[\alpha]$

c. $\neg \phi[\beta]^{11}$

${ }^{10}$ Este es para la tradición clásica el principio de cooperación y las máximas griceanas (Grice, 1975; Horn, 1972); para los neogriceanos, el principio Q (Levinson, 1983); para los relevantistas, el principio de relevancia óptima (Carston, 1998), entre otros. No es objeto de este estudio discutir cuál resulta un mejor marco teórico para explicar este tipo de inferencias.

$11 \neg \phi[\beta]$ es la versión débil de la IE. Si fuera el caso que el supuesto de competencia (Geurts, 2010) aplicara, podría derivarse la implicatura $\phi[\neg \beta]$, es decir, la versión fuerte, ya que $\phi[\neg \beta]$ entraña $\neg \phi[\beta]$, pero no al contrario. Sin embar- 
Donde (9a) es la escala relevante, (9b) el enunciado donde se predica algo del elemento débil de la escala y (9c) la IE. Nótese que la IE es la negación de la predicación del elemento más fuerte de la escala, lo cual implica algo crucial. Como puede verse en (9), la presencia del elemento escalar fuerte es indispensable para que la IE tenga lugar. En otras palabras, el elemento escalar fuerte tendría que estar presente o, por lo menos, en algún nivel activo en la mente del oyente en el momento de la derivación de la IE. Resultaría difícil entender cómo podría llegarse a una inferencia como (1b) o (2b) si en el oyente no se activaran elementos léxicos como todos o $y$. Sin embargo, este no es el caso para las implicaturas de cantidad, donde la inferencia no necesita surgir bajo una forma específica o con la presencia de un elemento léxico en particular. Es por ello que, en el caso de la IE, el elemento fuerte de la escala debe estar disponible para que pueda ser negado. Desde esta perspectiva, resultaría pertinente establecer la siguiente restricción:

(10) Restricción de acceso para derivar IE

$\neg \phi[\beta]$ podrá ser derivada sii emitido $\phi[\alpha]$, donde $\alpha \in\langle\alpha, \beta, \delta \ldots\rangle$, $\beta$ es accesible.

A partir de (10), se podría explicar por qué ejemplos como (8) son poco plausibles, en el sentido de que es improbable que una inferencia del tipo No vi a un siamés en la escalera surja porque la escala $<$ gato, siamés $>$ es poco relevante, como ya se mencionó, debido a que no cumple con la restricción en (10).

La pregunta que surge entonces es: dada una escala de Horn cualquiera, ¿cómo saber si su elemento fuerte se encuentra accesible en el momento en que se utiliza el elemento débil? Para responder esta cuestión se recurrió a un experimento con la finalidad

go, el fortalecimiento de implicaturas de cantidad no concierne a este estudio, aunque resultaría interesante saber si el acceso léxico también interfiere en este fenómeno. 
de medir la velocidad de acceso léxico al elemento escalar fuerte a partir de la exposición al elemento débil.

\subsection{Acceso léxico y efecto de primado}

Para saber si el elemento fuerte de una escala se activa en dichas condiciones, convendría encontrar evidencia de que el elemento débil facilita el acceso al fuerte. El acceso léxico ha sido un fenómeno ampliamente estudiado desde perspectivas tanto psicológicas como lingüísticas y se han desarrollado diversos paradigmas para medirlo. Se tiene bastante evidencia, además, de que cuando dos elementos léxicos están relacionados de alguna manera, la exposición a uno facilitará el acceso al otro. En específico, se sabe que esto ocurre cuando dos elementos están relacionados por asociación o semánticamente (Carr \& Dagenbach, 1990; Neely, 1991). Si el elemento débil y el elemento fuerte de una escala estuvieran fuertemente relacionados, el acceso al segundo debería ser más rápido a partir de la exposición al primero que a partir de la exposición a cualquier otro elemento relacionado. Generalmente, la manera de medirlo es a través del tiempo de reacción (TR), debido a que, si una entrada léxica $A$ exhibe un TR menor a partir de una entrada léxica $\mathrm{B}$ que a partir de una $\mathrm{C}, \mathrm{A}$ y $\mathrm{B}$, estarán mucho más intensamente relacionadas que A y C. Además, la presencia de A activará el acceso a B.

Una forma de medir el acceso léxico en términos de TR es a través de una tarea de decisión léxica con efecto de primado. A partir de dicha tarea puede saberse si la exposición a una entrada léxica efectivamente activa el acceso a otra diferente.

\section{Experimento}

Se recurrió a una tarea de decisión léxica con efecto de primado con el objetivo de saber qué activaba con más fuerza el acceso al elemento fuerte de una escala: el elemento débil o un elemento 
competidor relacionado. El elemento competidor relacionado elegido fue un antónimo. ${ }^{12}$ Para asegurarse de que la activación ocurría a un nivel inconsciente, se recurrió al efecto de primado enmascarado. Se sabe que a partir de este paradigma se obtienen efectos de primado sin que los sujetos sean capaces de reconocer la entrada léxica conscientemente (Holender, 1986). En el Cuadro 1 se muestran las 19 escalas adjetivales que fueron objeto del experimento.

Cuadro 1. Grupo de escalas utilizadas en la prueba

\begin{tabular}{|c|c|c|}
\hline \multicolumn{3}{|c|}{ ESCALAS ADJETIVALES } \\
\hline $\begin{array}{l}<\text { <bueno, excelente> } \\
\text { <bueno, perfecto> } \\
<\text { <cansado, exhausto> } \\
<\text { <laro, blanco> } \\
\text { <especial, único> } \\
\text { <río, helado> } \\
\text { <grande, enorme> }\end{array}$ & $\begin{array}{l}\text { <húmedo, mojado> } \\
\text { <inteligente, brillante> } \\
<\text { <malo, pésimo> } \\
<\text { malo, terrible> } \\
<0 \text { scuro, negro> } \\
<\text { parecido, igual }>\end{array}$ & 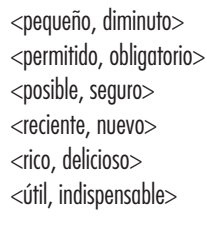 \\
\hline
\end{tabular}

\section{Materiales y método}

\subsection{Sujetos}

En el experimento participaron 60 hablantes nativos de español, todos estudiantes universitarios, procedentes de distintas carreras de la Universidad Nacional Autónoma de México. Los participantes fueron divididos en dos grupos: A y B. Para evitar en los sujetos un efecto de primado indeseado debido a la doble visualización de un mismo reactivo, el grupo A fue expuesto a una batería de reactivos críticos y controles distinta a la que fue expuesto el grupo B.

${ }^{12}$ Dado que hay pruebas empíricas que demuestran que no hay diferencia en cuanto al tiempo de reacción en el acceso entre pares sinónimos o antónimos (Hodgson, 1991; Perea \& Rosa, 2002), también pudo haberse elegido un sinónimo. 


\subsection{Materiales}

El experimento estuvo conformado por dos baterías de pruebas, A y B. Cada batería constó de 180 reactivos aleatorizados. El experimento fue programado en SuperLab. Los sujetos realizaron la tarea en una MacBook Pro utilizando el teclado de la computadora.

\subsection{Procedimiento}

La tarea consistía en que los participantes decidieran si la palabra que aparecía en la pantalla era del español o no. Al inicio de cada reactivo, y con el objetivo de fijar la mirada, los sujetos veían en el centro de la pantalla un signo de más (+) que desaparecía después de $500 \mathrm{~ms}$, y luego una máscara visual por otros $500 \mathrm{~ms}$. Inmediatamente aparecía el elemento primante en minúsculas, por $60 \mathrm{~ms}$, y después el objetivo en mayúsculas, el cual permanecía en la pantalla hasta que el participante decidiera si la palabra pertenecía al español o no, y oprimiera la tecla correspondiente, previamente programada con un color para cada caso: rojo para no y verde para sí (véase la Figura 4). Después de tomar la decisión, se desplegaba el siguiente reactivo y así sucesivamente.

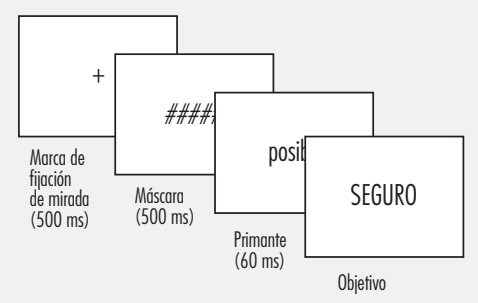

Figura 4. Pantallas presentadas por cada reactivo

\subsection{Reactivos}

La prueba constó de cinco tipos de reactivos, divididos en críticos, control y distractores. Los reactivos críticos estaban formados por 
los pares elemento débil/fuerte (D/F) y elemento débil/antónimo (D/A); los controles, por los pares pseudopalabra/elemento fuerte (C/F) y pseudopalabra/antónimo (C/A), ${ }^{13}$ y, finalmente, los distractores, por el par no-palabra/no-palabra. En el Cuadro 2 se muestra un ejemplo de cada reactivo y su proporción en cada prueba.

\begin{tabular}{|c|c|c|}
\hline TIPO DE REACTIVO & EJEMPLO PRIMANTE / OBJETIVO & ProporLIÓN \\
\hline Crítico escala (D/F) & posible / SEGURO & $15 \%$ \\
\hline Crítico competidor (D/A) & posible / IMPOSIBLE & $15 \%$ \\
\hline Control escala (c/F) & puevori / SEGURO & $15 \%$ \\
\hline Control competidor (c/A) & puevori / IMPOSIBLE & $15 \%$ \\
\hline Distractor & kleklex / KLEKLEX & $70 \%$ \\
\hline
\end{tabular}

Los reactivos se distribuyeron en dos grupos de tal forma que un mismo sujeto no fuera expuesto más de una vez al mismo primante, para evitar efectos indeseados. En el Cuadro 3 se muestra dicha distribución por grupo.

\begin{tabular}{lll}
\multicolumn{3}{l}{ Cuadro 3. Ejemplo de distribución de los reactivos por grupo } \\
\hline & Reactivos cRíticos & Reactivos conTROL \\
\hline Grupo A & posible / SEGURO & puevori/ IMPOSIBLE \\
Grupo B & posible / IMPOSIBLE & puevori / SEGURO \\
\hline
\end{tabular}

\subsection{Predicciones}

Al comparar los TR de los reactivos críticos - pares D/F vs D/A-, se esperaba encontrar que por lo menos en una parte de los 19 ca-

${ }^{13}$ Las pseudopalabras empezaban con la misma letra que el primante al cual controlaban y tenían la misma longitud. Ejemplo: primante malo, control mipo. En el Anexo puede consultarse la lista completa de reactivos críticos y competidores, así como de los controles. 
sos el reactivo D/F exhibiera TR menores en comparación con el reactivo D/A. Esto daría evidencia de que en este grupo se accedería más rápidamente al elemento fuerte a partir de la presentación del elemento débil. El acceso más rápido reflejaría una mayor disponibilidad del elemento más fuerte y por tanto una mayor relevancia de la escala misma. En el caso contrario, donde el reactivo competidor D/A exhibiera TR menores, el elemento fuerte estaría menos disponible y por tanto las escalas serían menos relevantes.

En el caso de los controles, se esperaba encontrar un comportamiento similar que se alineara con la predicción para los críticos, es decir, que los controles del grupo de críticos D/F con menores TR exhibieran por su parte también TR bajos y viceversa.

\section{Resultados y discusión}

\subsection{Resultados y discusión de las predicciones}

El análisis de los TR en el reconocimiento del elemento fuerte de la escala o del antónimo a partir del elemento débil como primante mostró que efectivamente hubo una diferencia, aunque no de la forma en la que se había predicho. A través de la prueba de distribución Wilcoxon test, se calculó primero la significatividad estadística ${ }^{14}$ de la diferencia en los TR en el reconocimiento del par D/F vs el par D/A. En el Cuadro 4 se muestran los resultados de esta diferencia en los casos donde hubo significatividad estadística.

\begin{tabular}{|c|c|}
\hline \multicolumn{2}{|c|}{ CASOS CON SIGNFICATIIIDAD EF US ANT } \\
\hline$<$ bueno, excelente> / malo & $p=0.031$ \\
\hline <grande, enorme > / pequeño & $p=0.022$ \\
\hline
\end{tabular}

${ }^{14}$ En lingüística, y en general en ciencias sociales, se acepta como límite de significación estadística de $p$ el valor de $\leq 0.050$, lo que significa que existe un $5 \%$ de probabilidad en contra de la hipótesis nula. 
(continuación)

Cuadro 4. Casos que exhibieron significatividad en la diferencia elemento débil / fuerte vs débil / antónimo

\begin{tabular}{|c|c|}
\hline \multicolumn{2}{|c|}{ CASOS CON SIGNFFCATIVIIDAD EF US ANT } \\
\hline$<$ malo, terrible $>$ / bueno & $p=0.013$ \\
\hline$<$ malo, pésimo> / bueno & $p=0.010$ \\
\hline$<$ <ico, delicioso > pobre & $p=0.009$ \\
\hline <húmedo, mojado> / seco & $p=0.006$ \\
\hline$<$ oscuro, negro $>$ / claro & $p=0.006$ \\
\hline$<$ bueno, perfecto> / malo & $p=0.003$ \\
\hline$<$ pequeño, diminuto> / adulto & $p=0.001$ \\
\hline
\end{tabular}

En estos 9 casos, el antónimo fue reconocido más rápidamente que el elemento fuerte a partir del elemento débil. Los casos que no mostraron significatividad estadística se agrupan en el Cuadro 5.

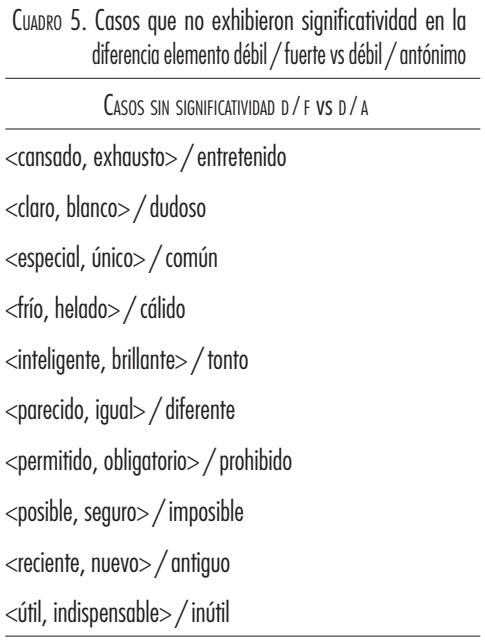

Como puede verse a partir de los dos cuadros, $47 \%$ exhibió una diferencia significativa en los TR. Es decir, en casi la mitad de los casos el antónimo de un elemento débil de una escala es recono- 
cido más rápidamente que el elemento fuerte. Sin embargo, en el restante $53 \%$ no existe una diferencia significativa, lo que sugiere que tanto el antónimo como el elemento fuerte son reconocidos con la misma velocidad. Esto, como se discute en los siguientes apartados, tiene implicaciones interesantes para la relación de escalaridad.

En cuanto a los reactivos control, se había predicho que se comportarían de forma similar a los críticos, es decir que para aquellos reactivos cuyos sujetos reportaran TR bajos, se encontraría que los reactivos control correspondientes producirían TR bajos y viceversa. De la misma forma que sucedió con los reactivos críticos, la predicción tampoco se cumplió para los controles. En el Cuadro 6 se muestra la diferencia en los TR entre el par D/F y el par C/F.

Cuadro 6. Casos que exhibieron significatividad en la diferencia elemento débil / fuerte vs pseudopalabra / elemento fuerte

\begin{tabular}{|c|c|}
\hline \multicolumn{2}{|c|}{ CASOS CON SIGNFICATIVIDAD SIGNFFCATIVIDAD D / F VS C/A } \\
\hline helida, húmedo / mojado, seco & $p=0.041$ \\
\hline puvemori, possible / seguro, imposible & $p=0.025$ \\
\hline bolmi, bueno / perfecto, malo & $p=0.016$ \\
\hline
\end{tabular}

Puede observarse que solo en $16 \%$ de los casos el elemento fuerte es reconocido más rápidamente a partir del control que a partir del elemento débil, lo que significa que para el restante $84 \%$ de los casos, no hubo una diferencia significativa en cuanto a TR en el reconocimiento del elemento fuerte, ya sea a partir del elemento débil o de un control. El caso contrario sucedió con la diferencia entre el par D/A y el par C/A. En el Cuadro 7, se muestra que en $68 \%$ de los casos el antónimo es reconocido más rápidamente a partir del elemento débil.

Esto implica que para el restante $32 \%$ de los casos no hubo una diferencia significativa en el reconocimiento del antónimo, ya sea a partir de un control o del elemento débil. 


\begin{tabular}{ll}
$\begin{array}{l}\text { Cuadro 7. Casos que exhibieron significatividad en la diferencia elemento } \\
\text { débil / antónimo vs pseudopalabra / antónimo }\end{array}$ \\
\hline \multicolumn{1}{c}{ Casos con significatividad o / A vs c / A } \\
\hline fica, frío / helado, cálido & $p=0.047$ \\
mipo, malo / terrible, bueno & $p=0.023$ \\
mipo, malo / pésimo, bueno & $p=0.023$ \\
rori, rico / delicioso, pobre & $p=0.017$ \\
gajofa, grande / enorme, pequeño & $p=0.012$ \\
helida, húmedo / mojado, seco & $p=0.011$ \\
parenco, parecido / igual, diferente & $p=0.011$ \\
bolmi, bueno / excelente, malo & $p=0.006$ \\
bolmi, bueno / perfecto, malo & $p=0.006$ \\
oncuma, oscuro / negro, claro & $p=0.003$ \\
roltregu, reciente / nuevo, aniguo & $p=0.003$ \\
ilinesimana, inteligente / brillante, tonto & $p=0.002$ \\
pomflasia, permitido / obligatorio, prohibido & $p=0.000$
\end{tabular}

\subsection{Discusión general}

Como se mostró en la sección anterior, ninguna de las predicciones se cumplió en el sentido de que, a partir del encuentro con un elemento débil de una escala, ningún elemento fuerte fue reconocido más rápidamente que el antónimo correspondiente. Sin embargo, esto no es tan sorprendente, pues los antónimos resultan un competidor muy fuerte. A pesar de ello, en $57 \%$ de los casos no se registró ninguna diferencia significativa en la velocidad de acceso entre ambos, lo que sugiere que en esta situación la exposición al elemento débil de la escala facilita el acceso al elemento fuerte mucho más que para el restante $43 \%$. Esto permite dividir la muestra total en dos grupos de escalas: por un lado, las que comparten elementos que facilitan su acceso entre sí y, por otro, las que no. En el Cuadro 8 se muestran los dos grupos. 
Cuadro 8. Casos donde los elementos de una escala facilitan el acceso entre sí y donde no lo hacen

\begin{tabular}{|c|c|}
\hline Se Facllita el AcCeso & No SE FaCLIITA EL ACCESO \\
\hline$<$ cansado, exhausto > & $<$ bueno, excelente> \\
\hline$<$ claro, blanco $>$ & $<$ bueno, perfecto> \\
\hline <especial, único> & <grande, enorme> \\
\hline <río, helado> & <húmedo, mojado> \\
\hline$<$ inteligente, brillante> & $<$ malo, pésimo $>$ \\
\hline <parecido, igual > & $<$ malo, terrible $>$ \\
\hline$<$ permitido, obligatorio > & $<0$ scuro, negro $>$ \\
\hline$<$ posible, seguro $>$ & $<$ pequeño, diminuto $>$ \\
\hline$<$ reciente, nuevo> & $<r i c 0$, delicioso $>$ \\
\hline$<$ <util, indispensable> & \\
\hline
\end{tabular}

Ahora bien, en cuanto a los casos particulares cuyo acceso es facilitado por el elemento débil, podemos ver que entre sí también exhiben diferencias. En la Figura 5 se muestra el diagrama de caja de estos 10 casos.

Lo que puede observarse al comparar el comportamiento de los sujetos al tomar la decisión léxica en estos 10 casos es que la mayoría de las escalas presenta valores extremos que van desde los $1000 \mathrm{~ms}$ hasta casi los $2500 \mathrm{~ms}$. Solo en <reciente, nuevo > la distribución de los valores es normal. Las diferencias en la distribución y en los valores extremos pueden deberse a varios factores, como la longitud de palabra (como en indispensable) o la frecuencia de uso (exhausto vs nuevo). También puede verse que para $40 \%$ la mediana no excede los $700 \mathrm{~ms}$, lo que sugiere que en estas escalas el elemento fuerte fue accedido más rápidamente que para el restante $60 \%$. Estos casos podrían tratarse de escalas todavía más relevantes que el resto.

Por otro lado, el análisis del comportamiento de los controles revela una característica muy interesante tanto de la relación de escalaridad como de la relación de antonimia. Al comparar los resultados obtenidos a partir de las diferencias entre D/F vs C/F, se 


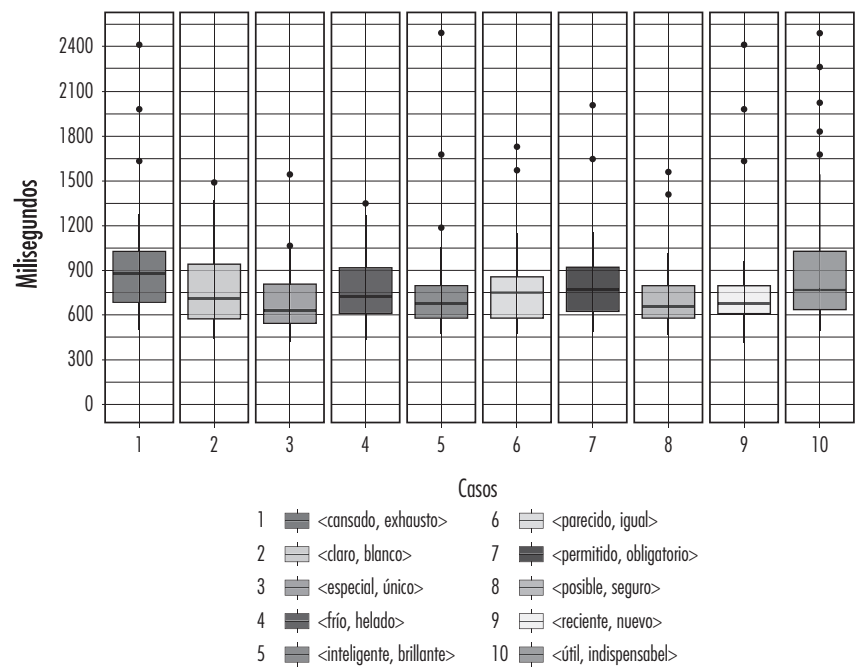

Figura 5. Tiempo de reacción de los casos que no exhibieron significatividad, es decir, del grupo de escalas cuyo acceso es facilitado por el elemento débil

observa que en ningún caso el elemento fuerte fue accedido más rápidamente a partir del débil que a partir de una pseudopalabra; al contrario, en 16\% de los casos la pseudopalabra facilitó el acceso al elemento fuerte, aunque este dato no es significativo. En general, esto sugiere que la relación de escalaridad (en el sentido de qué tanto un elemento débil activa el acceso a un elemento fuerte) es por sí misma muy débil, pues en la gran mayoría de los casos no existe una diferencia significativa al acceder a un elemento fuerte a partir de uno débil o de una pseudopalabra que inicia con la misma letra y tiene la misma extensión de palabra.

\section{Conclusiones}

Pese a que ninguno de los elementos fuertes de las escalas fue reconocido más rápidamente que los antónimos a partir de la exposición al elemento débil, como se esperaba que ocurriera, la diferencia hallada en los tiempos de reacción muestra dos cosas: 
1) los casos en los que hay significación estadística en cuanto a la diferencia en TR son evidencia de que los antónimos son accedidos más rápidamente que los elementos fuertes de las escalas, lo que indica que dichos elementos fuertes están menos disponibles que los antónimos, y 2) los casos en los cuales no hay significación estadística en cuanto a la diferencia en tiempos de TR indican que su acceso no difiere notablemente, lo que sugiere que los elementos fuertes están tan disponibles como los antónimos.

Esta divergencia en términos de acceso entre los casos agrupados en el Cuadro 8 podría representar también una diferencia en la tasa de derivación de IE. Si ocurre que, como se planteó antes, el acceso al elemento fuerte de la escala se vea facilitado a partir de la exposición al débil resulta un factor clave para la derivación de IE, se esperaría encontrar que las escalas pertenecientes al grupo de "Casos donde se facilita el acceso" (Cuadro 8) se comportaran como las escalas cuantificacionales o de conectivas, es decir que el uso de su elemento débil facultaría al oyente a derivar una IE. Un estudio empírico que pusiera a prueba la capacidad de estos dos grupos de escalas para generar la derivación de IE podría confirmar los hallazgos reportados en este artículo.

Los resultados también muestran que la relación de antonimia es mucho más fuerte que la relación de escalaridad, en el sentido de que se accede más rápidamente a un elemento léxico a partir de su antónimo que a partir de una pseudopalabra, en otros términos, la relación de antonimia favorece el primado entre pares. De manera opuesta, la relación de escalaridad no favorece el primado y en algunos casos lo dificulta. Probablemente se deba a que la relación de escalaridad pierde su propiedad de relación léxica cuando se invierte el orden del conjunto, ${ }^{15}$ mientras que la relación de an-

15 Recuérdese que las escalas de Horn son conjuntos ordenados cuyos elementos mantienen una relación de entrañamiento lógico que va del elemento fuerte al débil y no al contrario. En el experimento, esta relación (que asumo es una relación léxica como la de antonimia o sinonimia) se rompe al presentar a los participantes la escala en sentido contrario, es decir, como primante el elemento 
tonimia, al no depender de ningún orden, se mantiene constante sin importar cuál elemento del par funcione como primante.

A pesar de que los resultados muestran que efectivamente las escalas se comportan de forma distinta en cuanto a acceso léxico se refiere, este estudio explora la naturaleza léxica-semántica y no pragmática de la escalaridad. Convendría entonces examinar las relaciones que mantienen los elementos débiles de las escalas con otros elementos léxicos relacionados, a fin de obtener un panorama más completo de qué elementos (además de antónimos) son susceptibles de acceso léxico a partir del uso de un elemento débil de una escala.

Por otro lado, resulta patente que estudios como el presente solo abordan un aspecto del problema: la parte léxico-semántica involucrada en un fenómeno pragmático. Es evidente que una pregunta como "¿a partir del uso de qué escalas se deriva una IE?” no puede responderse solo con un estudio sobre acceso léxico; sin embargo, al ser parte del problema un fenómeno léxico en principio, parece razonable, al menos metodológicamente, abordar temas como el acceso léxico antes que la derivación misma de implicaturas.

\section{Referencias}

Carr, Thomas H., \& Dagenbach, Dale (1990). Semantic priming and repetition priming from masked words: Evidence for a center-surround attentional

débil y como objetivo el elemento fuerte. Una forma de preservar la relación léxica sería haber hecho lo opuesto, presentar a los participantes como primante el elemento fuerte y como objetivo el elemento débil. Aunque creo que en este caso los tiempos de reacción habrían bajado considerablemente para la mayoría de las escalas, creo también que no se estaría más que probando la relación lógica entre los elementos de los conjuntos ordenados y no el acceso al elemento más fuerte a partir de la exposición al más débil, como tendría que suceder al derivar una IE. 
mechanism in perceptual recognition. Journal of Experimental Psychology: Learning, Memory, \& Cognition, 16(2), 341-350.

Carston, Robrn (1998). Informativeness, relevance and scalar implicature. En Robyn Carston \& Seiji Uchida (Eds.), Relevance theory: Applications and implications (pp. 179-237). Ámsterdam: John Benjamins. ChierChia, Gennaro (2004). Scalar implicatures, polarity phenomena and the syntax/pragmatics interface. En Adriana Belletti (Ed.), Structures and beyond: The cartography of syntactic structures (Vol. 3, pp. 39103). Oxford: Oxford University Press.

Curcó Cobos, María, \& Peralta Estrada, María (2013). On the interpretation of numerals. Ponencia presentada en 13th International Pragmatics Conference, IPrA. Nueva Delhi, India.

GeUrTs, Bart (2010). Quantity implicatures. Cambridge: Cambridge University Press.

Grice, H. PAul (1975). Logic and conversation. En Peter Cole \& Jerry L. Morgan (Eds.), Syntax and semantics 3: Speech acts (pp. 41-58). Nueva York: Academic Press.

Hoogson, James M. (1991). Informational constraints on pre-lexical naming. Language and Cognitive Processes, 6(3), 169-205.

Horn, LAURENCE Robert (1972). On the semantic properties of logical operators in

English (Tesis doctoral inédita). Universidad de California en Los Ángeles, Los Ángeles.

Horv, Laurence Robert (1992). Pragmatics, implicature and presupposition. En W.

Bright (Ed.), International Encyclopedia of Linguistics (Vol. 3, pp. 260-266). Nueva York: Oxford University Press.

Horn, Laurence Robert (1996). Presupposition and implicature. En Shalom Lappin

(Ed.), The handbook of contemporary semantic theory (pp. 299319). Oxford: Blackwell Reference.

Holender, Danel (1986). Semantic activation without conscious identification in dichotic listening, parafoveal vision, and visual masking: a survey and appraisal. Behavioral and Brain Sciences, 9(1), 1-23.

Huang, Y I Ting; Spekke, Ellzabeth, \& Snedeker, Jesse (2013). What exactly do numbers mean?

Language Learning and Development, 9(2), 105-129.

Levinson, STepHen C. (1983). Pragmatics. Cambridge: Cambridge University Press. 
Neely, James (1991). Semantic priming effects in visual word recognition: A selective review of current findings and theories. En Derek Besner \& Glyn W. Humphreys (Eds.), Basic processes in reading: Visual word recognition (pp. 264-336). Hillsdale: Erlbaum.

Paparagou, Anna, \& Musolino, Julen (2003). Scalar implicatures: Experiments at the semantics-pragmatics interface. Cognition, 86(3), 253-282.

Perea, Manuel, \& Rosa, Eva (2002). Does the proportion of associatively related pairs modulate the associative priming effect at very brief stimulus-onset asynchronies? Acta Psychologica, 110(1), 103-124.

Sperber, Dan, \& Wilson, Derdore (1995). Relevance: Communication and cognition. Oxford: Blackwell.

van Tiel, Bob; van Mlltenburg, Emeli; Zevakhina, Natalla, \& Geurts, Bart (2016). Scalar diversity. Journal of Semantics, 33(1), 137-175.

VILLAEÑOR, Gala Karina (2017). Interpretación de términos numéricos: un estudio empírico. Estudios de Lingüística Aplicada, 35(66), 135-172.

Zevakhina, Natala (2012). Strength and similarity of scalar alternatives. En Ana Aguilar Guevara, Anna Chernilovskaya \& Rick Nouwen (Eds.), Proceedings of Sinn und Bedeutung 16 (Vol. 2, pp. 647-658). Utrecht: MIT Working Papers in Linguistics. 


\section{Anexo}

Lista completa de reactivos críticos, competidores y de control

\begin{tabular}{|c|c|c|c|}
\hline \multicolumn{2}{|c|}{ ELEMENTOS FUERTES } & \multicolumn{2}{|c|}{ ANTÓNIMOS } \\
\hline Primante & Objetivo & Primante & Objetivo \\
\hline bueno & excelente & bueno & malo \\
\hline bueno & perfecto & bueno & malo \\
\hline cansado & exhausto & cansado & entretenido \\
\hline claro & blanco & claro & dudoso \\
\hline especial & único & especial & común \\
\hline frío & helado & frío & cálido \\
\hline grande & enorme & grande & pequeño \\
\hline húmedo & mojado & húmedo & seco \\
\hline inteligente & brillante & inteligente & tonto \\
\hline malo & pésimo & malo & bueno \\
\hline malo & terrible & malo & bueno \\
\hline oscuro & negro & oscuro & claro \\
\hline parecido & igual & parecido & diferente \\
\hline pequeño & diminuto & pequeño & adulto \\
\hline permitido & obligatorio & permitido & prohibido \\
\hline posible & seguro & posible & imposible \\
\hline reciente & nuevo & reciente & antiguo \\
\hline rico & delicioso & rico & pobre \\
\hline útil & indispensable & útil & inútil \\
\hline
\end{tabular}

Estudios de Lingüistica Aplicada, año 37, número 70, diciembre de 2019, pp. 9-36

doi: 10.22201/enallt.01852647p.2019.70.869 
REACTIVOS CONTROL

\begin{tabular}{|c|c|c|c|}
\hline \multicolumn{2}{|c|}{ ELEMENTOS FUERTES } & \multicolumn{2}{|c|}{ ANTÓNIMOS } \\
\hline Primante & Objetivo & Primante & Objetivo \\
\hline bolmi & excelente & bolmi & malo \\
\hline bolmi & perfecto & bulmi & malo \\
\hline cragode & exhausto & cragode & entretenido \\
\hline cunje & blanco & cunje & dudoso \\
\hline entucire & único & entucire & común \\
\hline fica & helado & fica & cálido \\
\hline gajofa & enorme & gajofa & pequeño \\
\hline helida & mojado & helida & seco \\
\hline ilinesimana & brillante & ilinesimana & tonto \\
\hline mipo & pésimo & mipo & bueno \\
\hline mipo & terrible & mupo & bueno \\
\hline oncuma & negro & oncuma & claro \\
\hline puvemori & igual & puvemori & diferente \\
\hline pemfore & diminuto & pemfore & adulto \\
\hline pomflasia & obligatorio & pomflasia & prohibido \\
\hline parenco & seguro & parenco & imposible \\
\hline roltregu & nuevo & roltregu & antiguo \\
\hline rori & delicioso & rori & pobre \\
\hline ulje & indispensable & ulje & inútil \\
\hline
\end{tabular}

Estudios de Lingüistica Aplicada, año 37, número 70, diciembre de 2019, pp. 9-36

doi: 10.22201/enallt.01852647p.2019.70.869 